\title{
城市公园和郊区公园生物多样性评估的指标
}

\author{
陈 波 包志毅 \\ ( 浙江大学园艺系, 杭州 310029)
}

摘要: 随着城市化进程的加快, 城市的生物多样性不可避免地受到城市化的各种影响, 城市及其郊区的生物多样性 保护越来越受到人们的重视。城市公园与郊区公园中往往具有高度多样化的生境, 并保存着某些自然植被片段和 动物物种, 那里的生物多样性较高。可见, 在城市和郊区的生物多样性保护中, 公园生物多样性的保护是一个非常 关键的环节, 而对其生物多样性的评估又是有效保护的基础。目前, 我国生物多样性评估方面的研究工作多集中 于物种水平, 而对生境的研究较少, 但实践证明, 保护生境比保护物种更为重要。本文介绍了比利时学者 Hermy \& Cornelis 在比利时西佛兰德省的 Loppem 市立公园的保护实践中构建的一种对城市公园和郊区公园中的生物多样 性进行评估的方法。该方法从两个方面展开: 生境多样性和物种多样性。在生境水平上, 首先对各种生境单元进 行分类, 这些单元被分为面状、线状和点状要素。针对每种要素, 分别计算了 Shannon-Wiener 多样性指数和饱和度 指数。饱和度指数是实际的多样性指数与最大可能的多样性指数之比。在物种水平上, 使用了物种数、ShannonWiener 多样性指数和饱和度指数来评估公园中的高等植物、蝴蝶、两栖动物和饲养的鸟类等物种。这样, 就获得了 20 个生物多样性指标，根据这些指数就可以对 Loppem 市立公园内的生物多样性进行评估。结合我国生物多样性 评估工作的实际要求, 文章最后对上述方法进行了讨论, 指出该方法对我国公园的生物多样性评估工作具有借鉴 意义,但在运用时各地需要结合本地的实际情况。

关键词：生物多样性指标，公园，生境多样性，物种多样性

中图分类号:Q16,X176

文献标识码 : A

文章编号 : 1005-0094(2003)02-0169-08

\section{Indicators for monitoring biodiversity in urban and suburban parks}

CHEN Bo BAO Zhi-Yi

Department of Horticulture , Zhejiang University , Hangzhou 310029

Abstract : Urban and suburban parks are rich in habitats, plants and animals. With the rapid development of urbanization, biodiversity in cities and suburbs will decrease inevitably, so it is important to protect the biodiversity in parks. To achieve this goal, monitoring biodiversity is basic. Up to now, most studies on biodiversity conservation in China focus on species diversity, but few on habitat diversity. Actually, it has been proven that habitat conservation is more important than species conservation. Therefore, we introduce a method for the general monitoring of the biodiversity in urban and suburban parks developed by Hermy and Cornelis, two Belgian scholars. The method partitions diversity along two axes , habitat diversity and species diversity. At the habitat level, the diversity in so-called“ habitat units" is measured. These units are divided into planar, linear and punctual elements. For each category they calculated a Shannon-Wiener diversity index and a saturation index. The latter is the ratio of the actual diversity index to the theoretical maximum diversity index in the region. At the species level, species richness and biodiversity indices of vascular plants, butterflies, amphibians and breeding birds were calculated. These values were compared with the total number of species in Flanders, resulting in a saturation index for each park for plants and animals. In this way, 20 biodiversity indicators were obtained. This method was applied to measure the biodiversity in the municipal park of Loppem ( West Flanders , Belgium). We discuss the advantages and disadvantages of this method, and suggest that it should be utilized, based on specific situations. 
Key words : biodiversity indicator, park , habitat diversity , species diversity

\section{1 前言}

生物多样性是人类生存的基础。目前,人类对 自然的过度利用导致生物多样性的大量、快速丧失， 特别是随着全球范围城市化进程的加快，城市和郊 区的生物多样性不可避免地受到城市化的各种影 响。人类在城市建设的过程中，一方面破坏了动植 物栖息的自然生境,并建造了许多新的特化生境，另 一方面摒弃了大量原有的乡土物种, 又引进了许多 外来物种, 这必然导致城市及其郊区生物多样性的 严重丧失。中国目前正处在城市化高速发展的时 期, 因此城市及其郊区的生物多样性保护和研究在 我国更具有紧迫性和重要性。

公园是在城市化过程中幸存下来的或者是恢复 了的破碎的城市生境 (Fernández-Juricic \& Jokimoäki 2001)。公园中往往具有高度多样化的生 境 (如森林、灌丛、树篱、草地、牧场、草坪、花园、池 塘、建筑物、道路和小径等), 保存着某些自然植被 片段和多种动物, 那里的生物多样性往往非常高。 因此，保护城市公园和郊区公园的生物多样性是区 域生物多样性保护的重要环节。虽然在城市化进程 中郊区受到的干扰明显低于市区,但是市区和郊区 不仅在行政上，而且在生态环境和社会经济上也都 是一个整体(杨小波等,2000)，必须将市区与郊区 的生物多样性保护结合起来,才能实现对整个城市 生物多样性的有效保护。

生物多样性的评估是有效保护生物多样性、合 理利用其资源、保证其可持续发展的基础和关键。 因此，构建合理可行的生物多样性评估指标体系，对 生物多样性进行客观的评估, 不仅可以为人类对其 合理的经营利用提供科学的指导, 而且可以为国家 和国际水平的政策制定及贯彻实施提供更可靠的依 据。

\section{2 我国生物多样性评估的一般方法}

自从 1943 年 Fisher 首先提出物种多样性概念, 并运用 $\alpha 、 \beta 、 \gamma$ 指数来研究群落的物种多样性以来， 有关生物多样性的研究更加深入, 从定性研究逐步 走向定量化研究, 出现了 10 多种评估群落物种多样 性的指标, 如物种的丰富度 (richness)、多样性 (di- versity)、多度 (abundant)、均匀度 (evenness) 等 (曾 志新等,1999)。目前，我国生物多样性评估方面的 研究工作多集中于物种水平 (林涓等,1999;Primack 季维智, 2000;傅伯杰等,2001) ,有关物种生 存环境的评估方法尚不多见。而对生境甚至生态系 统的保护比物种保护更为重要( 陈灵芝,马克平, 2001; Gordon, 1993; Naveh, 1994; Noss , 1996) ,因为世 界上现存的大多数物种现在还不为人知, 而生境和 生态系统的保护可以同时保护生境内的所有物种及 其基因、物种和生态学过程。按照 Dramstad et al. (1996) 的观点, 物种多样性和生境多样性常常呈正 相关，这是因为多样化的生境可能包含更多适宜物 种生存的条件, 特别适宜那些多生境物种的生存。 生境多样性程度高,一方面引起大型斑块数目及斑 块内部物种数目的减少, 另一方面,引起斑块的边缘 生境面积的相对扩大以及边缘物种数目增大。即生 境多样性减少了稀有的内部种的丰度, 增加了边缘 种的丰度增加了要求两个以上景观单元物种的丰 度,同时提高了潜在的总物种的共存性。对于生境 多样性的评估方法, 国内已有学者开展了一些理论 上的研究(史作民等,1996;曾志新等,1999; 傅伯杰 等，2001），但由于某些指标的计算方法不统一，而 且所需的工作量较大, 故在实际工作中较难开展。 可见，我国有必要加强这方面的研究与实践，而借鉴 国外的先进方法与经验是必要的。

\section{3 比利时的生物多样性评估方法}

为了对城市公园和郊区公园中的生物多样性进 行监测并对由于管理或其他环境影响造成的公园结 构和物种组成的变化进行评估, 比利时学者 Hermy \& Cornelis (2000) 构建了一套对城市公园和郊区公 园中的生物多样性进行评估的方法, 并将该方法运 用到比利时西佛兰德省的 Loppem 市立公园的实践 中。生境多样性 ( habitat diversity) 与物种多样性 ( species diversity) 是评估生物多样性的有效指标, 而 Hermy 和 Cornelis 的方法正是从这两个方面开展 评估的。

\section{1 生境多样性评估}

为了便于对生境多样性进行评估, 可将生境分 为许多生境单元 (habitat unit)。通过实地的详细调 
查,可以列出佛兰德斯地区城市公园和郊区公园中 的所有生境单元的名单 (表 1 )。名单中的每个单元 都必须明确地加以定义，其描述不能有任何混淆。 该名单中只考虑了可能对生物多样性产生重要影响 的要素,而忽略了对生物多样性影响不大或无影响 的因素。由于不同类型的生境单元对公园中的生物 多样性具有不同的影响，并且测量的方法也不同，因 此可将所有的单元分为 3 种类型 : 点状要素、线状要 素和面状要素。面状要素以面积 $\left(\mathrm{m}^{2}\right)$ 表示, 线状要 素以长度 $(\mathrm{m})$ 表示, 点状要素则以数目 (个) 表示。 结合地理信息系统 (GIS) ,可以从数字地图中计算 出面状要素的面积、线状要素的长度和点状要素的 数目。

在表 1 所示的生境单元名单中包含 56 种生境 单元, 其中, 面状要素 32 种、线状要素 19 种、点状要 素 5 种。面状要素主要包括自然林分、人工林、草地 和园地、池塘和建筑物等类型; 而小路和各种类型的 树筃、路缘、水道和河岸则是重要的线状要素。由于 线状要素也具有一定的宽度,严格来说它们也属于 面状要素。因此，必须对它们进行严格的界定。一 般说来，当一个要素的长宽之比大于 10 时应作为线 状要素。点状要素包括单株树、水池、冰库以及一些 其他的基础设施。同样, 这些点状要素也占有面积, 因此必须限定点状要素的最大面积不应超过 100 $\mathrm{m}^{2}$, 当面积超过 $100 \mathrm{~m}^{2}$ 时上述要素就可以看作面状 要素。利用场地的信息和详细的航空照片, 可以测 定面状、线状和点状要素的属性。将其数字化之后, 就可以分别计算出它们的面积、长度和数量。根据 这些数据计算出城市公园和郊区公园中生境单元的 总面积、长度或数量的比例 (\%)。将这些比例代入 Shannon-Wiener 多样性指数 $\left(H^{\prime}\right)$ 计算公式, 即可计 算出生境单元的多样性 :

$$
H^{\prime}=-\sum_{i=1}^{s}\left(n_{i} / N\right) \ln \left(n_{i} / N\right)
$$

式中 , $i$ 是第 $i$ 个生境单元,$s$ 是生境单元的总数, $n_{i}$ 是生境单元 $i$ 的面积、长度或数量, $N$ 是公园中生境 单元的总面积、总长度或总数。

除了使用 Shannon-Wiener 多样性指数计算生境 多样性之外，还应计算生境的饱和度指数 (saturation index)。饱和度指数是实际测定的多样性指数和最 大可能的多样性指数之比 (\%), 即 : 饱和度指数 (S) $=H^{\prime} / H^{\prime}{ }_{\text {max }} \times 100 \%$ 。其中, $H^{\prime}{ }_{\text {max }}$ 可以通过下式计
算 :

$$
H_{\text {max }}^{\prime}=-\ln \frac{1}{S_{\text {max }}}=\ln S_{\text {max }}
$$

式中, $S_{\text {max }}$ 是生境单元的总数。

对一个既定的研究地区 (如本文中的佛兰德 斯) 的所有公园来说, $H^{\prime}{ }_{\text {max }}$ 是恒定不变的。通过上 式可以计算出佛兰德斯地区公园中面状、线状和点 状要素的 $H^{\prime}{ }_{\text {max }}$ 值分别为 3.47、2.94 和 1.61。

所有生境单元的总体饱和度指数 $\left(S_{t}\right)$ 可以通过 3 种生境单元类型的 3 个饱和度指数进行加权平均 来计算。其公式为 :

$$
S_{t}=\frac{S_{p l} n_{p l}+S_{l i} n_{l i}+S_{p u} n_{p u}}{n_{t}}
$$

式中, $S_{p l}$ 是面状要素的饱和度指数, $n_{p l}$ 是面状要素 的数量; $S_{l i}$ 是线状要素的饱和度指数, $n_{l i}$ 是线状要素 的数量 $; S_{p u}$ 是点状要素的饱和度指数, $n_{p u}$ 是点状要 素的数量 $; n$ 是生境单元的总数。

\section{2 物种多样性评估}

\subsection{1 物种群体的选择}

Hermy \& Cornelis 选择了 4 个不同的物种群体 来测定公园的生物多样性。它们是维管植物 (包括 本地种、移植种和外来种, 以及品种和变种) 、蝴蝶、 乌类和两栖动物。选择这些物种群体的首要原因在 于公众和决策者对它们都非常熟悉。

选择植物的原因在于植物构建了城市公园的结 构, 并且为动物提供了多样化的生境。同时, 传统的 城市公园常常具有高度的生物价值和园艺价值。但 由于大多数植物的寿命相对较长, 它们对环境变化 的反应往往有时滞效应。

研究中使用蝴蝶是因为它们的生命较为短暂, 对生境结构和植物组成的变化很敏感。因此, 它们 具有相对较高的生境专一性, 对它们进行评估相对 容易。

由于水对公园的建设以及公园内的各物种的生 存具有十分重要的作用, 因此, 研究中包括了池塘。 通过研究池塘中两栖动物的生活方式, 可以将公园 中陆地与水中的生境质量相结合。像蝴蝶一样, 两 栖动物对环境胁迫因子也是非常敏感的。

由于乌类能够反映城市公园的许多特征 (例如 安静程度、植物结构、树龄、管理水平、生境异质性 等)，同时，人们对它们都非常熟悉，不会出现鉴定 上的困难, 因此研究中也包含了鸟类。研究群体中 
表 1 佛兰德斯城市公园和郊区公园的生境单元分类名单 (Hermy \& Cornelis, 2000)

Table 1 List of habitat units distinguished in urban and suburban parks in Flanders, Belgium

\section{1 面状要素 Planar elements}

1.1 自然林分 :由天然或半天然森林植被组成的单元 Forest stand : unit composed of a more or less natural forest vegetation

1.1 .1 落叶树林 落叶树林分 Deciduous wood : forest stand of deciduous trees

1.1 .1 .1 矮林: :定期修剪的灌木层林分 Coppice : forest stand of regularly cutted thickets (1)

1.1 .1 .2 标准的矮林: 定期修剪的灌木层和上层乔木林分 Coppice with standards : forest stand of regularly cutted thickets and upper trees (2)

1.1.1.3 公园树林:具有下层丛林的孤立木林分 Park wood : forest stand of single trees with ligneous undergrowth (3)

1.1.1.4 高度整齐的阔叶林: 高度整齐的落叶林林分 Leafy, regular high forest : forest stand of regular high deciduous trees (4)

1.1 .2 针叶林: 针叶林林分 Coniferous wood: forest stand of conifers (5)

1.1 .3 混交林: 落叶和针叶林林分 Mixed wood : forest stand of deciduous and coniferous trees (6)

\section{2 种植园 :由人工种植的树木组成的单元 Plantation : unit composed of planted trees}

1.2.1 果园 : 种植果树的封闭单元 Orchard : enclosed unit planted with fruit trees (7)

1.2.2 林中草地 种植了林木的草地 Forest grassland : grassland planted with forest trees (8)

1.2.3 树廊: 不具有下木的线状人工林 Tree gallery : linear plantation of trees without undergrowth (9)

1.2.4 树木园 种植了不同种类的树木并具有教育功能的人工林 Arboretum : plantation of different tree species with an educational function (10)

1.2.5人工林:由低于 $3 \mathrm{~m}$ 的林木组成的人工林 Forest plantation : plantation of forest trees $(<3 \mathrm{~m})(11)$

1.3 迷宫:由封闭的树篱以迷宫的形式组成的单元 Labyrinth : unit composed of close hedges in labyrinth form (12)

1.4 灌木林:由灌木组成的单元 Shrub plantation : unit composed of shrubs (13)

1.5 草地 :由草本植物组成的单元 Grassland : unit composed of grass species

1.5.1 草坪 经常修剪的草地 Lawn : frequently mown grassland (14)

1.5 .2 体育场 经常修剪且作为运动场的草地 Sports field : frequently mown grassland used as sports ground (15)

1.5 .3 干草地 :用来制造干草的草地 Hay meadow : grassland used to make hay (16)

1.5 .4 牧场 :放牧的草地 Pasture : grassland grazed by animals (17)

1.5.5 干草牧场: 制造干草之后放牧的草地 Hay-pasture : grassland that is grazed after hay-making (18)

1.6 高大的草本植被 :由天然的草本植物包括芦苇类植被组成的单元 Tall herb vegetation : unit composed of rough herbs, inclusive reed vegetation (19)

1.7 欧石南丛生的荒野:由矮灌木组成的单元 Heathland : unit composed of dwarf shrubs (20)

1.8 农业地区 :由农作物组成的单元 Agricultural area : unit composed of arable crops (21)

1.9 休耕地 :由休耕的土地组成的临时性单元 Fallow land : temporary unit composed of fallow ground (22)

1.10 园地:由蔬菜、水果或观赏植物组成的封闭性单元 Garden : enclosed unit composed of vegetables, fruit or ornamental plants

1.10 .1 菜园 :由蔬菜和水果组成的园地 Kitchen garden : garden composed of vegetables and fruit (23)

1.10 .2 药草园 :由药草组成的园地 Herb garden : garden composed of medicinal herbs (24)

1.10 .3 蓄薇园 :由蓄薇属植物组成的园地 Rose garden : garden composed of roses (25)

1.10.4 观赏植物园 :由其他的观赏植物组成的园地 Ornamental garden : garden composed of other ornamental plants (26)

1.11 观赏植物林:由观赏植物组成的非封闭单元 Ornamental plantation : non-enclosed unit composed of ornamental plants (27)

1.12 水文要素:由水组成的单元 Water feature : unit composed of water

1.12.1 城堡的护城河 环绕历史建筑的水文要素 Castle-moat : water feature round a historical building (28)

1.12 .2 池塘 没有任何建筑的水文要素 Pond : water feature free from each building (29)

1.13 建筑:由建筑物包括建筑之间的有限空间组成的单元 Building : unit composed of buildings, inclusive the limited space between the buildings (30)

1.14 停车场:由停靠车辆的场所组成的单元 Car park : unit composed of parking places for vehicles

1.14.1半硬化的停车场 没有完全硬化的停车场 Half-hardened : parking with a hardening that is not completely sealed (31) 
表 1 (续) Table 1 ( continued)

1.14.2 未硬化的停车场 没有进行任何硬化的停车场 Not hardened : parking without any hardening (32)

2 线状要素 Linear elements

2.1 小路：道路两旁各具有一排或四排树木，包括边缘 Alley：double or four-double row of trees, including the verges (33)

2.2 树列 : 列植的树木 Tree row : row of trees (34)

2.3 树篱 线状木质植被 Hedge : linear wooden vegetation

2.3.1 修剪过的树筃 经过定期修剪的树篱 Sheared hedge : hedge that is regularly sheared (35)

2.3.2 未修剪的树篱 没有经过修剪的树筃 Non-sheared hedge : hedge that is not sheared (36)

2.3.3 树木堤坝: 在堤坝上人工栽植的树筃 Wooden embankment : hedge on an embankment created by humans (37)

2.4 路缘 沿着道路的未硬化条带 Road verge : non-hardened strip along a road (38)

2.5 堤岸 :水道或水文要素两旁的条形陆地 Bank : strip of land on each side of a water feature or a watercourse

2.5.1 水文要素的堤岸 护城河或池塘的堤岸 Bank of a water feature : bank of a castle-moat or pond

2.5.1.1 自然式: 没有经过人工加固的堤岸 Natural : bank not consolidated by humans (39)

2.5.1.2 半自然式:由人工加固的, 可能还有植被的堤岸 Semi-natural : bank consolidated by humans where vegetation is still possible (40)

2.5.2 水道的堤岸沟渠、小溪或河流的堤岸 Bank of a watercourse : bank of a ditch, brook or river

2.5.2.1 自然式没有经过人工加固的堤岸 Natural : bank not consolidated by humans (41)

2.5.2.2 半自然式:由人工加固的, 可能还有植被的堤岸 Semi-natural : bank consolidated by humans where vegetation is still possible (42)

2.6 水道 :用来排水的线状要素 Watercourse : linear element used for the discharge of water

2.6.1 沟渠: 宽度小于 $1 \mathrm{~m}$, 可能有水的水道 Ditch : watercourse with a width of max. $1 \mathrm{~m}$ that may contain water (43)

2.6.2 小溪: 宽度小于 $3 \mathrm{~m}$, 常年有水的水道 Brook : watercourse with a width of max. $3 \mathrm{~m}$ that always contains water (44)

2.6.3 河流: :宽度大于 $3 \mathrm{~m}$ 的水道 River : watercourse with a width of $>3 \mathrm{~m}(45)$

2.7 道路基础设施 : 为行人和车辆服务的带状设施 Road infrastructure : strip used and prepared for pedestrians and service traffic

2.7.1 道路: :宽度大于 $2 \mathrm{~m}$ 的道路基础设施 Road : road infrastructure with a width of $>2 \mathrm{~m}$

2.7.1.1 半硬化的 没有完全硬化的道路 Half-hardened : road with a hardening that is not completely sealed (46)

2.7.1.2 未硬化的 没有进行任何硬化的道路 Not hardened：road without any hardening (47)

2.7.2 凹陷的道路: 凹陷的道路基础设施及其边缘 Sunken road : sunken road infrastructure, including the verges (48)

2.7.3 小道: 宽度小于 $2 \mathrm{~m}$ 的道路基础设施 Path : road infrastructure with a width of $<2 \mathrm{~m}$

2.7.3.1 半硬化的 没有完全硬化的小道 Half-hardened : path with a hardening that is not completely sealed (49)

2.7.3.2 未硬化的 没有进行任何硬化的小道 Not hardened：path without any hardening (50)

2.8 墙壁 :用来围合空间的线状砖石建筑 Wall : linear masonry used as enclosing (51)

3 点状要素 Punctual elements

3.1 单株乔木或灌木:周围没有其他乔木或灌木围绕的乔木或灌木 Single tree or shrub : tree or shrub not surrounded by other trees or shrubs (52)

3.2 水池 :小而浅的死水, 面积 $\leqslant 100 \mathrm{~m}^{2}$ Pool : small , shallow, stagnant water $\leqslant 100 \mathrm{~m}^{2}$ (53)

3.3 冰库: 保存冰的库房 Icehouse : house where ice was kept (54)

3.4 坟墓 坟墓土堆 Tumulus : burial mound (55)

3.5 基础设施要素: 人工建筑 (井、喷泉、亭子、小教堂、纪念碑、雕像、桥梁、鸟舍……) Infrastructure element : human construction (well, fountain, kiosk, chapel, monument, statue, bridge, aviary, $\cdots$ ) (56)

注 : 1 56 表示 56 种生境单元 Note: The numbers $1 \sim 56$ represents 56 habitat units

包含了许多位于食物链顶端的种类 ( 如猫头鹰) 以 及候鸟等。

\subsection{2 植物种类}

为了测定公园中的植物多样性, 可采取对面状 和线状生境单元分层随机取样的方法。对草本植 物, 可选取 $4 \mathrm{~m}^{2}$ 的样方, 对乔木和灌木(高于 1.30 
$\mathrm{m}$ ) 可选取 $100 \mathrm{~m}^{2}$ 的样方。在每个样方中，列出所 有现存的维管植物种类, 并估计其盖度（\%);对于 墙面的攀缘植物, 其盖度用垂直盖度 $(\%)$ 表示。

由于物种丰富度和多样性通常随着面积的增加 而增加, 因此, 样方的大小应与公园总面积成比例。 在每个生境单元内随机取样, 样方的形状根据要素 的类型不同而不同。由于许多草本植物种类的花期 很短, 因此, 草本植物的名单要调查两次:一次在春 季, 另一次在夏季。在两个季节的调查结果中, 挑选 各种植物的多样性较高的结果进行计算。所有植物 种类的多样性指数 $\left(H_{p}\right)$ 可以通过乔灌木的多样性 指数与草本植物的多样性指数进行加权平均算出：

$$
H_{p}=\frac{H_{t r} n_{t r}+H_{h e} n_{h e}}{n_{t o t}}
$$

式中, $H_{t r}$ 是乔灌木的多样性指数, $n_{t r}$ 是木本植物样 方的数量; $H_{h e}$ 是草本植物的多样性指数, $n_{h e}$ 是草本 植物样方的数量; $n_{t o t}$ 是样方的总数。

\subsection{3 动物种类}

蝴蝶的物种数量用两种方法来测量。第一, 在 进行植物调查时所有看见的蝴蝶都作了记录; 第二, 在所有样方内专门进行蝴蝶物种数量的调查。这必 须在晴朗而暖和的日子进行, 因为蝴蝶在下雨或气 温低于 $20^{\circ} \mathrm{C}$ 时不会出现。

为了测定乌类的物种数量, 他们使用了现有的 数据并通过当地鸟类学者或自然团体对这些数据进 行了修正和补充。用同样的方法可以测定两栖动物 的物种数量。标准的取样过程包括设陷阱和布网。 最后将观察到的每个物种的物种数量与佛兰德斯地 区的物种总数进行比较, 从而计算出该地的饱和度 指数。

\section{4 比利时 Loppem 市立公园的生物多样性 评估}

为了验证上述方法的可行性 ,Hermy \& Cornelis 将其运用到 Loppem 市立公园。该公园位于比利时 西佛兰德省的布鲁日 (Bruges) 以南约 $10 \mathrm{~km}$ 处,占 地面积 $25 \mathrm{hm}^{2}$, 是一个物种丰富的新哥特式城堡公 园。

在进行生境单元测定时，他们在面状要素中选 取了 25 个 $100 \mathrm{~m}^{2}$ 的样方来测定公园中的木本植 物, 选取了 125 个 $4 \mathrm{~m}^{2}$ 的样方 (其中 50 个线状要素 和 75 个面状要素) 来测定草本植物。在进行物种
群体测定时，他们发现虽然佛兰德斯的外来植物种 类的数量非常高, 但具体数目不清楚, 因此,他们没 有计算饱和度指数。由于动物具有活动性, 其调查 过程比植物种类更难且更耗费时间。因此, 不能测 定动物的种群大小, 所以他们只调查了物种数量, 而 没有计算多样性指数，结果见表 2 。在研究中,由于 资金和研究水平的限制，仅能对一小部分生境和物 种多样性进行测量。但是, 可以将对部分物种进行 的多样性测定结果类推到其他未被测定的物种上， 从而使问题得以简化。

\section{5 讨论}

(1) 为了对城市公园和郊区公园的生物多样性 进行评估，上述方法分别从生境水平和物种水平上 进行了研究。物种多样性是生物多样性的直接体 现，科学地认识并描述公园中现有的生物物种，搞清 楚生物物种数量, 是公园生物多样性研究最基本也 是最关键的一环, 对生物多样性研究结果的综合性、 可靠性有着决定性的影响（陈灵芝等，2001）。生境 是各种生物的栖息地，生境条件与生物多样性息息 相关。为了实现物种的长期保存, 不仅要考虑目标 物种本身，还要考虑它所在的生态系统以及有关的 生态过程 (Franklin ,1993)。

（2）上述方法在评估物种多样性和生境多样性 时主要计算了 Shannon-Wiener 多样性指数和饱和度 指数。选择 Shannon-Wiener 多样性指数是因为它的 应用很普遍，人们对它也很熟悉;而饱和度指数可以 反映一个地区实际的生物多样性水平与理想状态下 生物多样性水平的差距，从而为生物多样性保护与 管理提供可靠的依据。当然，研究的目的不同，选取 的具体指标也可以不同。

(3) 物种多样性的评估主要针对各种植物、蝴 蝶、两栖动物和鸟类, 主要原因是由于人们对它们比 较熟悉, 或者是由于它们对环境变化很敏感。

(4) 对生境单元进行分类是生境多样性评估的 关键。一般来说, 在不同的情况下, 生境单元分类的 标准和方法应有所不同。上述分类方法虽然在很大 程度上受比利时国情的限制，但我国也可参考佛兰 德斯地区的生境分类方法并结合实际情况，对各地 区的生境单元进行分类研究。

一般地，对具体区域的生境类型分类包括 3 个 步骤:首先是根据遥感影像 (航、卫片) 解译，结合地 
表 2 Loppem 市立公园的生物多样性指标( Hermy \& Cornelis , 2000)

Table 2 Biodiversity indicators in the municipal park of Loppem

\begin{tabular}{|c|c|c|c|}
\hline $\begin{array}{l}\text { 生境单元 } \\
\text { Habitat unit }\end{array}$ & $\begin{array}{l}\text { 分类数 } \\
\text { No. of categories }\end{array}$ & $\begin{array}{l}\text { 多样性指数 } \\
\text { Diversity index }\end{array}$ & $\begin{array}{l}\text { 饱和度指数 (与公园中最大多样性相比) } \\
\text { Saturation index (compared to the maximum diversity in parks) }\end{array}$ \\
\hline $\begin{array}{l}\text { 面状要素 }(\text { 最大值 }=32 \text { ) } \\
\text { Planar elements }(\max .=32)\end{array}$ & 14 & 1.75 & $50.4 \%$ \\
\hline $\begin{array}{l}\text { 线状要素 }(\text { 最大值 = 19) } \\
\text { Linear elements }(\max .=19) \\
\text { 点状要素 }(\text { 最大值 }=5)\end{array}$ & 13 & 2.15 & $73.0 \%$ \\
\hline $\begin{array}{l}\text { Punctual elements }(\max .= \\
5)\end{array}$ & 4 & 0.68 & $42.0 \%$ \\
\hline $\begin{array}{l}\text { 总计 }(\text { 最大值 }=56) \\
\text { Total }(\max .=56)\end{array}$ & 31 & $1.78^{*}$ & $58.8 \% *$ \\
\hline $\begin{array}{l}\text { 物种群体 } \\
\text { Species groups }\end{array}$ & $\begin{array}{c}\text { 物种数量 } \\
\text { No. of species }\end{array}$ & $\begin{array}{c}\text { 多样性指数 } \\
\text { Diversity index }\end{array}$ & $\begin{array}{c}\text { 饱和度指数 (与佛兰德斯的物种数相比) } \\
\text { Saturation index ( compared to the number of species in Flanders) }\end{array}$ \\
\hline $\begin{array}{l}\text { 维管植物 } \\
\text { Vascular plants }\end{array}$ & 134 & 3.23 & - \\
\hline $\begin{array}{l}\text { 蝴蝶 }(\text { 最大值 }=80) \\
\text { Butterflies }(\max .=80)\end{array}$ & 9 & - & $11.2 \%$ \\
\hline $\begin{array}{l}\text { 两栖动物 }(\text { 最大值 }=13) \\
\text { Amphibians }(\max .=13)\end{array}$ & 3 & - & $23.1 \%$ \\
\hline $\begin{array}{l}\text { 饲养鸟类 (最大值 }=169) \\
\text { Breeding birds }(\text { max. }=169)\end{array}$ & 36 & - & $21.3 \%$ \\
\hline
\end{tabular}

＊加权平均值 Weighted average " - " 表示没有计算 Date not calculated

形图和其他图形及文字资料，加上野外调查结果，选 取并确定区域景观生态分类的主导要素和依据，初 步确定个体单元的范围及类型，构建初步的分类体 系。第二步, 详细分析各类单元的定性和定量指标， 表列各种特征。通过聚类分析确定分类结果，逻辑 序化分类体系。第三步，依据类型单元指标，经由判 别分析，确定不同单元的功能归属，作为功能性分类 结果。

初始分类的主要指标，一是地貌形态及其界线; 二是地表覆被状况，包括植被和土地利用等。区域 不同,生境单元分异要素就不相同, 类型特征指标中 选择的内容就应有所区别。一般包括地形、海拔、坡 向、坡度、坡形、地表物质、构造基础、 $\mathrm{pH}$ 值、土层厚 度、有机质含量、剥蚀侵蚀强度、植被类型及其覆盖 率、土地利用、区位指数、气温、降水量、径流指数、干 燥度、土壤主要营养成分含量以及管理集约程度等 等(傅伯杰等 2001)。

(5) 在公园中选取样地进行评估是上述方法的 特点之一, 这样做的好处在于节省人力、物力和财 力。通过一个相对有限的调查, 测定出一系列不同 层次上的公园生物多样性指标, 可以将对部分物种 进行的多样性测定结果类推到其他未被测定的物种 上。这样，该方法就可以运用到所有城市和郊区的
公园中。如果再将不同的公园中所得到的结果进行 比较, 我们就能够了解不同生境多样性与物种多样 性之间的关系。这样, 各个公园的生物多样性情况 就一目了然了。

(6) 上述方法也存在着一些不足,需要在实践 中加以改进。例如，该方法仅仅对高等植物和部分 动物进行了研究, 尚未涉及低等植物 (如地衣和苔 藓)、微生物以及其他动物, 不同公园的优势种、特 有种和濒危种都不同，应根据具体情况具体分析。 其次, 该方法只计算了公园生态系统中的结构多样 性 (包括物种多样性和生境多样性)，对一些重要的 生态过程如物种流、能量流和物质流都未进行研究。 因为对于公园生态系统而言, 生物的结构多样性和 功能多样性两个方面的内容都极为重要, 而生态过 程正体现了生物多样性功能方面的内容, 因此也应 对其进行评估。

\section{6 结语}

城市公园和郊区公园的生物多样性评估是复杂 和综合性的工作，国内很少开展，也无详细报道。它 涉及生物多样性的各个层次以及千变万化的环境和 管理条件，同时也受评估人的知识水平、专业经验和 对评估对象熟知程度的限制，尤其是指标体系的选 
取及其数量化更是因评估目的的不同而存在较大的 差别, 还必须在实践中不断加以完善。但有一点可 以肯定, 对城市公园和郊区公园生物多样性的评估 可以从两个方面着手:生境多样性和物种多样性。 上述方法较系统地为我们阐述了城市公园和郊区公 园中的生物多样性的评估过程和指标体系, 可操作 性和可靠性较强，值得我们在实际工作中灵活借鉴。

\section{参考文献}

Chen L-Z (陈灵芝). 1993. Status and Strategies for Biodiversity Conservation in China (中国的生物多样性现状及其保护 对策). Science Press, Beijing. (in Chinese)

Chen L-Z(陈灵芝) and Ma K-P(马克平)。2001. Biodiversity Science: Principles and Practices (生物多样性科学: 原理 与实践). Shanghai Science and Technology Press, Shanghai (in Chinese)

Dramstad W. E., Olson J. D. and Forman R. T. T. 1996. Landscape Ecology Principles in Landscape Architecture and Land-use Planning. Harvard University Graduate School of Design/Island Press, Cambridge.

Fernández-Juricic E. and Jokimäki J. 2001. A habitat island approach to conserving birds in urban landscapes: case studies from southern and northern Europe. Biodiversity and Conservation, 10(12): $2023 \sim 2043$.

Franklin J. F. 1993. Preserving biodiversity: species, ecosystems, or landscapes? Ecological Applications, 3(2) : 202 205.

Fu B-J(傅伯杰), Chen L-D (陈利顶), Ma K-M (马克明), Wang Y-L(王仰麟)。2001。Landscape Ecology, Theory and Application (景观生态学原理及应用). Science
Press, Beijing. (in Chinese)

Gordon H. 1993. Endangered at what level? Ecological Application, 3(2): $206 \sim 208$.

Hermy M. and Cornelis J. 2000. Towards a monitoring method and a number of multifaceted and hierarchical biodiversity indicators for urban and suburban parks. Landscape and Urban Planning, 49 (3 4): $149 \sim 162$.

Lin J. (林涓), Tang L-J(唐礼俊), Qian J. (钱吉). 1999. Urban development and biodiversity conservation. Shanghai Environmental Sciences (上海环境科学), 18(4)：188～ 189. (in Chinese)

Naveh Z. 1994. From biodiversity to ecodiversity: a landscape ecology approach to conservation and restoration. Research Ecology, 2(3): $180 \sim 189$.

Noss R. F. 1996. Ecosystem as conservation targets. TREE, 11 (8) : 351 .

Primack R. and Ji W-Z(季维智). 2000. A Primer of Conservation Biology (保护生物学基础). China Forestry Publishing House, Beijing. (in Chinese)

Shi Z-M(史作民), Cheng R-M(程瑞梅), Chen L(陈力), Liu S-R(刘世荣)。1996. Study on method for regional ecosystem biodiversity assessment. Rural Eco-environment (农 村生态环境), 12(2)：1５. (in Chinese)

Yang X-B(杨小波)，Wu Q-S(吴庆书)，Zou W(邹伟)，Luo C-Y(罗长英). 2000. Urban Ecology (城市生态学). Science Press, Beijing. (in Chinese)

Zeng Z-X(曾志新), Luo J(罗军), Yan L-H(颜立红), Zou JW(邹建文), Xiao L-T( 肖绿田). 1999. Study on indices and criteria for biodiversity assessment. Journal of Hunan Forestry Science \& Technology (湖南林业科技), 26(2): $26 \sim 29$. (in Chinese) 